\title{
Gene Transfer In Vivo with DNA-Liposome Complexes: Lack of Autoimmunity and Gonadal Localization
}

\author{
ELIZABETH G. NABEL, DAVID GORDON, ZHI-YONG YANG, LING XU, HONG SAN, \\ GREGORY E. PLAUTZ, BEI-YUE WU, XIANG GAO, ${ }^{1}$ LEAF HUANG,${ }^{1}$ and GARY J. NABEL
}

\begin{abstract}
Direct gene transfer into localized arterial segments can be performed in vivo by transfection with DNAliposome complexes. This technique holds promise for the treatment of human diseases, including malignancy and cardiovascular disorders. We have previously characterized the potential toxicity of this form of treatment in mice in vivo (Stewart et al., 1992). In this report, we examine two issues relevant to long-term expression of foreign recombinant genes: (i) the potential for autoimmune damage to major organs and (ii) DNA localization in gonadal tissue. Autoimmunity and toxicity of allogeneic major histocompatibility (MHC) gene transfer was assessed in mice after induction of an immune response to a recombinant murine class I MHC gene by direct gene transfer in vivo. Histological examination of brain, heart, lung, liver, kidney, spleen, and skeletal muscle revealed no clinically significant immunopathology or organ damage. The toxicity of gene delivery by DNA liposomes was also analyzed in pigs and rabbits in vivo. No histopathology was observed following the introduction of plasmids encoding several different gene products, and analysis of serum following DNA liposome delivery revealed no abnormalities of serum biochemical parameters. The potential for transfer of recombinant DNA into testes and ovary in animals was evaluated by the polymerase chain reaction. Although evidence of recombinant plasmid was consistently observed in transfected, but not untransfected, arterial sites and occasionally in lung, kidney, spleen, and liver, no plasmid DNA was detected in testes or ovary. These studies suggest that uptake of recombinant DNA following direct gene transfer by liposomal transfection in major organs is not associated with autoimmunity, toxicity, or gonadal localization. This method of direct gene transfer is therefore appropriate for human gene therapy.
\end{abstract}

\section{OVERVIEW SUMMARY}

The use of DNA-liposome complexes to transfer genes in vivo is now underway in an active clinical protocol. Mouse safety and acute toxicity data have been published previously (Hum. Gene Ther. 3, 267-275). In the present paper, Nabel et al. begin to provide long-term safety data, specifically with regard to the potential for autoimmune damage to major organs or for the transfer of the foreign genes into reproductive tissue.

\section{INTRODUCTION}

$\mathbf{T}$ HE DIRECT TRANSMISSION of recombinant DNA into specific organs in vivo for therapeutic purposes offers several attractive features for the treatment of human disease. Ex vivo gene therapy requires the removal of tissue from patients, establishment of cell lines in the laboratory, and delivery to the patient. These steps can be cumbersome, complex, and costly. By the direct administration of genes in vivo, many of these steps can be eliminated, simplifying procedures and reducing

Departments of Internal Medicine, Biological Chemistry, and Pathology, Howard Hughes Medical Institute, University of Michigan Medical Center, Ann Arbor, MI 48109-0688.

'Department of Pharmacology, University of Pittsburgh School of Medicine, Pittsburgh, PA 15261. 
delays in the initiation of treatment. Although direct gene transfer is attractive in some respects, several concerns remain regarding its potential toxicity. We have previously documented the lack of acute toxicity using DNA-liposome complexes in mice (Stewart et al., 1992). In this report, we address three issues relevant to potential chronic toxicities that may arise from this treatment: (i) immunopathology caused by autoimmunity, (ii) long-term organ toxicity, and (iii) DNA uptake by gonadal tissue that could increase the potential for inadvertent germ-line transfer.

Two methods have proven successful for arterial gene transfer in vivo, including infection with replication-defective retroviral vectors and transfection with DNA-liposome complexes (Nabel et al., 1989, 1990, 1992a; Lim et al., 1991). Retroviral vectors used in several direct gene transfer studies in vivo show no evidence for the generation of helper virus (Nabel et al., 1990). The safety of murine amphotropic replication-defective retroviral vectors has also been examined in a variety of experimental animal studies (Cornetta et al., 1990, 1991) and in recent human clinical trials (Rosenberg et al., 1990) using $e x$ vivo gene therapy. Recombinant DNA and cationic liposome complexes (Felgner et al., 1987; Felgner and Rhodes, 1991; Gao and Huang, 1991) have recently been developed for direct gene transfer in vivo (Nabel et al., 1990, 1992a; Stewart et al., 1992). Although these studies have demonstrated efficacy of gene transfer in experimental animals in vivo, the long-term sequelae of recombinant gene expression are not entirely known. The purpose of this report is to summarize safety and toxicity data generated from studies of DNA liposome-mediated gene transfer in vivo in several small and large animal models, including mice, rabbits, and pigs. Major organs were examined for recombinant gene expression, autoimmunity, toxicity, and biochemical abnormalities, and localization of recombinant DNA to germ line tissue was analyzed. Based on these studies, there are no apparent contraindications to this form of gene delivery for gene therapy.

\section{MATERIALS AND METHODS}

\section{Plasmids}

Plasmids containing recombinant human genes were prepared in eukaryotic plasmid expression vectors for liposomal transfection in vivo. These plasmid expression vectors included: $\mathrm{pLJ}$ encoding the Escherichia coli $\beta$-galactosidase gene (Price et al., 1987); pLJ encoding the human factor IX gene (Yao et al., 1991); pSVL encoding the human platelet-derived growth factor (PDGF) B (v-sis) gene (generously provided by Dr. Brent Cochran); pMEX encoding the human acidic fibroblast growth factor (aFGF) gene (kindly provided by Dr. Thomas Maciag); pRKS encoding the human transforming growth factor- $\beta$ (TGF- $\beta$ )) gene (Arrick et al., 1992); pMTE encoding the human transforming growth factor- $\alpha$ (TGF- $\alpha$ ) (kindly provided by Dr. Rik Derynck); pLJ encoding the human class I major histocompatability complex (MHC) gene, HLA-B7 (Nabel et al., 1992a); and pLJ encoding the murine class I MHC gene, H-2K $\mathrm{K}^{\text {S }}$ (Plautz et al., 1992).

\section{Transfection with DNA-liposome complexes}

In these studies, we have used two formulations of cationic liposomes: Lipofectin (Bethesda Research Laboratories, Gaithersburg, MD) (Felgner et al., 1987) and DC-cholesterol (Gao and Huang, 1991). All mice were transduced with DC-cholesterol liposomes $(n=17)$, and all rabbits with Lipofectin $(n=27)$. Pigs were transduced with Lipofectin $(n=39)$ or with DC-cholesterol $(n=1)$.

Previous studies had established conditions for optimal transfection. We have found that the ratio of DNA to lipid and the absolute concentration of each affect the transfection efficiency in endothelial cells (Stewart et al., 1992), and these conditions were used for the present study. DNA-liposome complexes were prepared for in vivo transfection, as previously described, using $5 \mu \mathrm{l}$ of Lipofectin and $2-5 \mu \mathrm{g}$ of plasmid DNA $(\geqslant 1$ $\mathrm{mg} / \mathrm{ml}$ ) (Nabel et al., 1992a) or $15 \mathrm{n} M$ cationic DC-cholesterol liposomes and $1 \mu \mathrm{g}$ of plasmid DNA (Gao and Huang, 1991; Stewart et al., 1992).

\section{Direct gene transfer in vivo}

DNA-liposome complexes were introduced into Yorkshire pigs or New Zealand white rabbits by intravenous injection or arterial transfection. Direct arterial transfection was performed as previously described in peripheral iliofemoral arteries of pigs using a catheter (Nabel et al., 1990, 1992a). Rabbit arterial transfections were performed by surgically exposing the distal aorta and bilateral iliofemoral arteries. Temporary ligatures were placed around the distal aorta and right femoral artery, isolating a 2-cm length of artery. Side branches were tied off and a 1.5-mm angioplasty catheter (Scimed, Minneapolis, MN) was placed and inflated in the proximal iliac artery through a distal aorta arteriotomy, creating a $1-\mathrm{cm}$ protected length of artery between the proximal catheter tip and the distal ligature for transfection. The balloon was inflated, occluding the proximal portion of the artery and the artery was rinsed through the distal tip of the catheter with $3 \mathrm{cc}$ of saline and $3 \mathrm{cc}$ of OptiMEM (Bethesda Research Laboratories). The distal ligature was tightened around the right iliofemoral artery, creating a $1-\mathrm{cm}$ protected length of artery from the tip of the catheter to the ligature, and $0.1-0.2 \mathrm{ml}$ of transfection solution was instilled into the $1-\mathrm{cm}$ arterial space. Following $20 \mathrm{~min}$ of transfection, the balloon was deflated, the distal ligature was removed, and the catheter was withdrawn. The arteriotomy was repaired and circulation was restored. Intravenous injections were made into rabbit ear central vein.

Studies of chronic organ toxicity and autoimmunity in mice were performed in 17 adult female mice (strain BALB/c) by intravenous, intraperitoneal, and subcutaneous injections of DNA-liposomes complexes. Animals were divided into two groups based upon the generation of cytolytic T cells to $\mathrm{H}-2 \mathrm{~K}^{\mathrm{S}}$ : immune and nonimmune groups. Immune animals $(n=10)$ were injected with $\mathrm{RSV}-\mathrm{H}-2 \mathrm{~K}^{\mathrm{S}}$ plasmid under the following conditions (i) intravenous injection $(n=7)$, four injections and sacrificed at 75 days $(n=4)$, and three injections and sacrificed at 28 days $(n=3)$; (ii) subcutaneous injection $\times 3$ and sacrificed at 28 days $(n=1)$; and (iii) intraperitoneal injection $\times 3$ 
Table 1. Analysis of Potential Chronic Organ Toxicity or Autoimmunity in Mice InJeCted WITH H-2K $\mathrm{K}^{\mathrm{S}}$ DNA LIPOSOMES

\begin{tabular}{|c|c|c|c|c|c|c|c|c|}
\hline \multicolumn{4}{|c|}{ CTL immunity } & & \multicolumn{2}{|c|}{ Pathology } & \multicolumn{2}{|c|}{$P C R$} \\
\hline $\begin{array}{l}\text { Nonimmune } \\
(n=6)\end{array}$ & & $\begin{array}{l}\operatorname{Im} n \\
(n=\end{array}$ & & & $\begin{array}{c}\text { Nonimmune } \\
(n=7)\end{array}$ & $\begin{array}{l}\text { Immune } \\
(n=10)\end{array}$ & $\begin{array}{c}\text { Nonimmune } \\
(n=7)\end{array}$ & $\begin{array}{l}\text { Immune } \\
(n=10)\end{array}$ \\
\hline $\begin{array}{l}\mathrm{CT} 26 \mathrm{~K}^{\mathrm{S}} \\
2.6 \pm 0.6\end{array}$ & $\begin{array}{c}\text { CT26 } \\
1.6 \pm 0.4\end{array}$ & $\begin{array}{c}\text { CT26 K } \\
20.6 \pm 6.6\end{array}$ & $\begin{array}{c}\text { CT26 } \\
1.6 \pm 0.3\end{array}$ & $\begin{array}{l}\text { Brain } \\
\text { Heart } \\
\text { Lung } \\
\text { Liver } \\
\text { Kidney } \\
\text { Ovary }\end{array}$ & $\begin{array}{l}\text { Normal } \\
\text { Normal } \\
\text { Normal }^{\mathrm{a}} \\
\text { Normal }^{\mathrm{a}} \\
\text { Normal } \\
\text { Normal }\end{array}$ & $\begin{array}{l}\text { Normal } \\
\text { Normal } \\
\text { Normal } \\
\text { Normal }^{\mathrm{a}} \\
\text { Normal } \\
\text { Normal }\end{array}$ & $\begin{array}{l}0 / 10 \\
1 / 10 \\
4 / 10 \\
0 / 10 \\
0 / 10 \\
0 / 10\end{array}$ & $\begin{array}{l}2 / 7 \\
2 / 7 \\
1 / 7 \\
1 / 7 \\
1 / 7 \\
0 / 7\end{array}$ \\
\hline
\end{tabular}

Immune response to $\mathrm{H}-2 \mathrm{~K}^{\mathrm{S}}$ (percent lysis) at a 50:1 effector to target ratio, organ pathology, and presence of DNA by PCR is shown.

${ }^{a}$ Incidental pathological findings of no clinical significance.

and sacrificed at 28 days $(n=2)$. Nonimmune animals $(n=7)$ were injected with Rous sarcoma virus (RSV) $\beta$-galactosidase $(n=4)$ by subcutaneous $(n=2)$, intraperitoneal $(n=1)$, or intramuscular $(n=1)$ routes and sacrificed at 28 days; RSV HLA-B7 $(n=2)$ by intravenous route and sacrificed at 64 and 74 days; and $\mathrm{K}^{\mathrm{s}}$ alone by subcutaneous $(n=1)$ delivery and sacrificed at 28 days. For all studies, the mice received $1.25 \mu \mathrm{g}$ of plasmid DNA and $7.5 \mu$ l of DC-cholesterol prepared in a final volume of $0.7 \mathrm{ml}$ of lactated Ringer's, with $0.1 \mathrm{ml}$ injected. Serological testing for mouse hepatitis virus were performed using an ELISA and an immunofluorescence assay (Charles River Laboratories, New Bedford, MA).

\section{Lymphocyte preparation and cytolytic T-cell assay}

Murine splenic lymphocytes were incubated in RPMI containing $10 \%$ fetal bovine serum, penicillin $(50 \mathrm{U} / \mathrm{ml})$, streptomycin $(50 \mu \mathrm{g} / \mathrm{ml})$, L-glutamine $(2 \mathrm{mM})$, and 2-mercaptoethanol $\left(5 \times 10^{-5} M\right)$ at a concentration of $10^{6} / \mathrm{ml}$ on a layer of irradiated CT26 H-2 $\mathrm{K}^{\mathrm{S}}$. stimulator cells for 5 days. Target cells were incubated with ${ }^{51} \mathrm{Cr}(100 \mu \mathrm{Ci})$ at $37^{\circ} \mathrm{C}$ for $2 \mathrm{hr}$, washed $\times 3$, and plated in 96-well microtiter plates at $10^{4}$ cells per well (Romano, 1986). Supernatant was replaced with prestimulated effector cells $\left(3 \times 10^{4}\right.$ to $7.5 \times 10^{5}$ cells) suspended in $100 \mu \mathrm{l}$ of RPMI containing $10 \%$ FBS, penicillin/streptomycin, L-glutamine, and 2-mercaptoethanol. Plates were centrifuged at $(100 \times g)$ for $5 \mathrm{~min}$ at the beginning and end of the assay to settle lymphocytes. Supernatant $(50 \mu \mathrm{l})$ from the reaction was counted in a Beckman gamma counter.

\section{Analysis of recombinant gene expression}

The expression of the human recombinant genes was analyzed up to 126 days following direct gene transfer. Genomic DNA was prepared from transfected and nontransfected arteries, ovary, testes, heart, lung, liver, spleen, kidney, and skele-

Table 2. Histological Analysis of Tissues Following Introduction of DNA Liposomes IN Vivo

\begin{tabular}{|c|c|c|c|c|}
\hline & \multicolumn{2}{|c|}{ Pigs } & \multicolumn{2}{|c|}{ Rabbits } \\
\hline & $\begin{array}{l}\text { DNA liposomes } \\
\quad(n=28)\end{array}$ & $\begin{array}{l}\text { Normal controls } \\
\quad(n=6)\end{array}$ & $\begin{array}{l}\text { DNA liposomes } \\
\quad(n=22)\end{array}$ & $\begin{array}{c}\text { Normal controls } \\
\quad(n=2)\end{array}$ \\
\hline $\begin{array}{l}\text { Heart } \\
\text { Lung }\end{array}$ & Normal & Normal & Normal & Normal \\
\hline $\begin{array}{l}\text { Focal peribronchial lymphoid } \\
\text { aggregates }\end{array}$ & $11 / 28$ & $4 / 6$ & $12 / 22$ & $2 / 2$ \\
\hline \multicolumn{5}{|l|}{ Liver } \\
\hline $\begin{array}{l}\text { Focal portal mononuclear } \\
\text { inflammatory aggregates } \\
\text { Kidney }\end{array}$ & $10 / 28$ & $2 / 6$ & $4 / 22$ & $1 / 2$ \\
\hline Focal tubular stasis & $1 / 28$ & $0 / 6$ & $0 / 22$ & $0 / 2$ \\
\hline $\begin{array}{l}\text { Focal interstitial mononuclear } \\
\text { inflammatory cells }\end{array}$ & $2 / 28$ & $0 / 6$ & $0 / 22$ & $0 / 2$ \\
\hline \multicolumn{5}{|l|}{ Spleen } \\
\hline Congestion & $5 / 28$ & $2 / 6$ & $0 / 22$ & $0 / 2$ \\
\hline Skeletal muscle & Normal & Normal & Normal & Normal \\
\hline Nontransfected artery & Normal & Normal & Normal & Normal \\
\hline \multicolumn{2}{|l|}{ Gonadal tissue } & Normal $(5 / 5)$ & Normal $(10 / 10)$ & Normal (2/2) \\
\hline $\begin{array}{l}\text { Ovary } \\
\text { Testes }\end{array}$ & $\begin{array}{l}\text { Normal }(20 / 20) \\
\text { Normal }(2 / 2)\end{array}$ & Normal $(1 / 1)$ & Normal $(12 / 12)$ & \\
\hline
\end{tabular}


Table 3. Evaluation of Serum Biochemical Parameters after In Vivo Gene Transfer in Pigs

\begin{tabular}{|c|c|c|c|c|c|c|}
\hline & \multicolumn{4}{|c|}{ Plasmid DNA } & \multirow[b]{2}{*}{$\begin{array}{l}\text { Saline control } \\
\quad(n=5)\end{array}$} & \multirow[b]{2}{*}{$\begin{array}{c}\text { Normal range } \\
\quad(n=7)\end{array}$} \\
\hline & $\begin{array}{c}0-7 \text { days } \\
(n=8)\end{array}$ & $\begin{array}{c}7-21 \text { days } \\
(n=2 I)\end{array}$ & $\begin{array}{c}22-67 \text { days } \\
(n=4)\end{array}$ & $\begin{array}{l}\text { Summary } \\
0-67 \text { days } \\
(n=28)\end{array}$ & & \\
\hline Albumin (grams/dl) & $3.0 \pm 0.1$ & $3.1 \pm 0.1$ & $2.4 \pm 0.0$ & $2.9 \pm 0.7$ & $3.1 \pm 0.2$ & $3.1 \pm 0.1$ \\
\hline Alk Phos (IU/liter) & $274 \pm 31$ & $236 \pm 13$ & $319 \pm 9$ & $266 \pm 13$ & $275 \pm 52$ & $309 \pm 21$ \\
\hline Amylase (units/liter) & $2381 \pm 156$ & $2449 \pm 153$ & $2315 \pm 267$ & $2312 \pm 81$ & $1918 \pm 139$ & $2424 \pm 180$ \\
\hline Bilirubin (mg/dl) & $0.1 \pm 0.1$ & $0.2 \pm 0.0$ & $0.3 \pm 0.1$ & $0.2 \pm 0.1$ & $0.1 \pm 0.0$ & $0.3 \pm 0.1$ \\
\hline BUN (mg/dl) & $9 \pm 1$ & $10 \pm 1$ & $8 \pm 1$ & $10 \pm 1$ & $8 \pm 1.3$ & $11 \pm 1$ \\
\hline Calcium (mg/dl) & $10.0 \pm 0.4$ & $9.3 \pm 0.1$ & $8.8 \pm 0.1$ & $9.4 \pm 0.2$ & $10.0 \pm 0.2$ & $9.8 \pm 0.2$ \\
\hline Chloride (mEq/liter) & $106 \pm 2$ & $105 \pm 1$ & $101 \pm 2$ & $105 \pm 1$ & $106 \pm 2$ & $108 \pm 12$ \\
\hline Cholesterol (mg/dl) & $103 \pm 6$ & $108 \pm 4$ & N/A & $115 \pm 10$ & $86 \pm 8$ & $95 \pm 12$ \\
\hline Creatinine $(\mathrm{mg} / \mathrm{dl})$ & $1.0 \pm 0.1$ & $1.0 \pm 0.0$ & $0.9 \pm 0.0$ & $1.0 \pm 0.1$ & $1.0 \pm 0.1$ & $1.0 \pm 0.1$ \\
\hline GGT (IU/liter) & $32 \pm 4$ & $33 \pm 2$ & $45 \pm 3$ & $32 \pm 2$ & $32 \pm 3$ & $34 \pm 6$ \\
\hline Globulin (total grams/dl) & $1.7 \pm 0.1$ & $2.0 \pm 0.1$ & $2.1 \pm 0.1$ & $1.9 \pm 0.1$ & $1.9 \pm 0.1$ & $2.0 \pm 0.2$ \\
\hline Glucose (mg/dl) & $156 \pm 16$ & $106 \pm 10$ & $164 \pm 11$ & $132 \pm 9$ & $126 \pm 34$ & $106 \pm 9$ \\
\hline LDH (IU/liter) & $384 \pm 27$ & $352 \pm 21$ & $372 \pm 4$ & $370 \pm 15$ & $371 \pm 53$ & $592 \pm 38$ \\
\hline Phosphorus (mg/dl) & $8.4 \pm 0.5$ & $8.4 \pm 0.2$ & $7.3 \pm 0.3$ & $8.1 \pm 0.2$ & $8.8 \pm 0.8$ & $8.8 \pm 0.4$ \\
\hline Potassium (mEq/liter) & $4.0 \pm 0.1$ & $4.0 \pm 0.1$ & $4.0 \pm 0.1$ & $4.0 \pm 0.1$ & $4.0 \pm 0.2$ & $4.6 \pm 0.4$ \\
\hline SGOT (IU/liter) & $22.3 \pm 3$ & $28.0 \pm 3$ & $35.0 \pm 3$ & $28 \pm 2$ & $26 \pm 3$ & $46 \pm 5$ \\
\hline SGPT (IU/liter) & $33 \pm 2$ & $42.0 \pm 3$ & $42.3 \pm 5$ & $38 \pm 2$ & $33 \pm 3$ & $36 \pm 4$ \\
\hline Sodium (mEq/liter) & $140 \pm 1$ & $140 \pm 1$ & $137 \pm 2$ & $139 \pm 1$ & $143 \pm 2$ & $145 \pm 2$ \\
\hline Total protein (grams/dl) & $4.9 \pm 0.2$ & $5.1 \pm 0.1$ & $4.5 \pm 0.1$ & $4.9 \pm 0.1$ & $5 \pm 0.2$ & $5.2 \pm 0.1$ \\
\hline Triglycerides (mg/dl) & $32.4 \pm 4$ & $27.4 \pm 3$ & $52 \pm 16$ & $34 \pm 4$ & $22 \pm 3$ & $64 \pm 15$ \\
\hline Uric acid (mg/dl) & 0.0 & 0.0 & 0.0 & 0.0 & 0.0 & 0.0 \\
\hline
\end{tabular}

Pigs were transduced in the iliofemoral artery with DNA-liposome complexes $(n=28)$ or saline $(n=5)$. Blood samples were obtained prior to sacrifice, and serum enzyme and chemistry values were analyzed (Roche Biomedical Laboratory, Dublin, $\mathrm{OH}$ ). A normal range of values was determined on pigs at the University of Michigan who had not undergone experimental manipulation. Mean values, SEM, and the number of subjects (in parentheses) are shown.

tal muscle by standard proteinase $\mathrm{K}$ digestion and phenol and chloroform extraction conditions. Oligonucleotide primers were synthesized to generate $\sim 350$-bp fragments, as follows: $E$. coli $\beta$-galactosidase, human HLA-B7, human factor IX, and murine $\mathrm{H}-2 \mathrm{~K}^{\mathrm{s}}$ genes as previously described (Nabel et al., 1990, 1992a; Yao et al., 1991; Stewart et al., 1992, respectively). PDGF B gene, sense TAC TCC TCT TAA GCT GCG TAT TCG G, antisense ACA CCA GGA AGT TGG CAT TGG TGC G; acidic FGF gene, sense CAA ACT CCT CTA CTG TAG CAA CGG G, antisense TTG CTT TCT GGC CAT AGT GAG TCC G; TGF- $\beta$ gene, sense TCC ACC TGC AAG ACT ATC GAC ATG G, antisense CAG CCG GTT GTC GAG GTA TCG CCA G; TGF- $\alpha$ gene, sense TGG TCC CCT CGG CTG GAC AGC TCG C, anti-sense TCG GAC CTG GCA GCA GTG TAT CAG C. Polymerase chain reaction (PCR) analysis was performed using $100 \mu \mathrm{g}$ of genomic DNA for 30 cycles of denaturation $\left(94^{\circ} \mathrm{C}, 1 \mathrm{~min}\right)$, annealing $\left(60^{\circ} \mathrm{C}, 2 \mathrm{~min}\right)$, and polymerization $\left(72^{\circ} \mathrm{C}, 2 \mathrm{~min}\right)$ in an automated DNA thermal cycler (Perkins-Elmer/Cetus). Samples were analyzed by ethidium bromide staining in $1 \%$ agarose gel. Genomic DNA from transfected cells was used as positive control $(1 \mu \mathrm{g})$. The sensitivity of detection has been estimated to be between 1 copy per $10^{3}$ to $10^{5}$ genomes.

\section{Analysis of organ toxicity}

Organ specimens including transfected and nontransfected artery, ovary or testes, brain, heart, lung, liver, spleen, kidney, and muscle were obtained at animal sacrifice, formalin-fixed, paraffin-embedded, and stained in hematoxylin and eosin. Representative sections were examined by an experienced pathologist (D.G.) in a blinded fashion.

Serum from rabbits and pigs was obtained prior to sacrifice, frozen at $-20^{\circ} \mathrm{C}$, and biochemical analyses were performed (Roche Biomedical Laboratories, Dublin, $\mathrm{OH}$ ). Mean values and SEM are expressed. Values from experimental groups were compared to sham-operated saline controls and normal animals that did not undergo any experimental procedure, using analysis of variance with Bonferroni correction. Results were considered statistically significant if $p \leqslant 0.05$.

\section{RESULTS}

\section{Lack of immune pathology after foreign MHC gene transfer}

An important question regarding the long-term toxicity from expression of a foreign histocompatibility gene introduced by direct gene transfer in vivo has been the possibility of inducing autoimmunity. In this study, we addressed this question by examining murine tissues following confirmation that a specific immune response to a foreign human class I MHC gene had been elicited. The presence and distribution of this plasmid DNA within different tissues was confirmed by PCR and was found consistently in murine liver, lung, and heart as previously noted (Stewart et al., 1992). 
Table 4. Evaluation of Serum Biochemical Parameters after IN Vivo Gene Transfer in Rabbits

\begin{tabular}{|c|c|c|c|c|c|c|}
\hline & \multicolumn{4}{|c|}{ Plasmid DNA } & \multirow[b]{2}{*}{$\begin{array}{l}\text { Saline control } \\
\quad(n=6)\end{array}$} & \multirow[b]{2}{*}{$\begin{array}{c}\text { Normal range } \\
\quad(n=6)\end{array}$} \\
\hline & $\begin{array}{c}0-7 \text { days } \\
(n=8)\end{array}$ & $\begin{array}{c}7-21 \text { days } \\
(n=3)\end{array}$ & $\begin{array}{c}22-I 20 \text { days } \\
(n=6)\end{array}$ & $\begin{array}{c}\text { Summary } \\
0-120 \text { days } \\
(n=17)\end{array}$ & & \\
\hline Albumin (grams/dl) & $3.3 \pm 0.1$ & $4.1 \pm 0.1$ & $3.7 \pm 0.1$ & $3.6 \pm 0.1$ & $3.7 \pm 0.1$ & $3.9 \pm 0.1$ \\
\hline Alk Phos (IU/liter) & $107 \pm 7$ & $50 \pm 11$ & $58 \pm 9$ & $80 \pm 8$ & $131 \pm 27$ & $140 \pm 30$ \\
\hline Amylase (units/liter) & $306 \pm 22$ & $303 \pm 14$ & $410 \pm 31$ & $342 \pm 19$ & $302 \pm 26$ & $351 \pm 29$ \\
\hline Bilirubin (mg/dl) & $0.2 \pm 0.0$ & $0.3 \pm 0.0$ & $0.3 \pm 0.0$ & $0.3 \pm 0.1$ & $0.2 \pm 0.0$ & $0.2 \pm 0.0$ \\
\hline BUN (mg/dl) & $15 \pm 1$ & $15 \pm 1$ & $19 \pm 1$ & $16 \pm 1$ & $13.2 \pm 1.4$ & $13 \pm 1$ \\
\hline Calcium (mg/dl) & $11.9 \pm 0.2$ & $11.7 \pm 0.1$ & $13.8 \pm 0.2$ & $12.5 \pm 0.3$ & $12.2 \pm 0.4$ & $12.4 \pm 0.1$ \\
\hline Chloride (mEq/liter) & $100 \pm 2$ & $107 \pm 1$ & $108 \pm 1$ & $104 \pm 1$ & $105 \pm 2$ & $104 \pm 1$ \\
\hline Cholesterol (mg/dl) & $49 \pm 2$ & $43 \pm 5$ & $33 \pm 2$ & $42 \pm 2$ & $56 \pm 7$ & $62 \pm 10$ \\
\hline Creatinine (mg/dl) & $0.8 \pm 0.0$ & $1.3 \pm 0.6$ & $1.0 \pm 0.0$ & $1.0 \pm 0.1$ & $1.1 \pm 0.1$ & $1.0 \pm 0.0$ \\
\hline GGT (IU/liter) & $3.9 \pm 0.6$ & $9.3 \pm 1.3$ & $10 \pm 1.0$ & $7 \pm 1$ & $5.3 \pm 1.1$ & $5.7 \pm 0.4$ \\
\hline Globulin (total grams/dl) & $1.4 \pm 0.1$ & $2.3 \pm 0.9$ & $1.4 \pm 0.0$ & $1.3 \pm 0.1$ & $1.5 \pm 0.1$ & $1.7 \pm 0.1$ \\
\hline Glucose (mg/dl) & $102 \pm 5$ & $156 \pm 9$ & $127 \pm 9$ & $120 \pm 6$ & $190 \pm 11$ & $102 \pm 7.2$ \\
\hline LDH (IU/liter) & $364 \pm 23$ & $242 \pm 42$ & $184 \pm 67$ & $279 \pm 33$ & $295 \pm 70$ & $386 \pm 71$ \\
\hline Phosphorus (mg/dl) & $4.5 \pm 0.1$ & $4.7 \pm 0.6$ & $3.4 \pm 0.2$ & $4.2 \pm 0.2$ & $7.3 \pm 0.7$ & $6.9 \pm 0.7$ \\
\hline Potassium (mEq/liter) & $4.5 \pm 0.2$ & $4.0 \pm 0.2$ & $5.3 \pm 0.4$ & $4.7 \pm 0.2$ & $4.0 \pm 0.2$ & $4.2 \pm 0.2$ \\
\hline SGOT (IU/liter) & $50 \pm 6$ & $17 \pm 2$ & $27 \pm 6$ & $36 \pm 5$ & $36 \pm 11$ & $26 \pm 4$ \\
\hline SGPT (IU/liter) & $50 \pm 4$ & $25 \pm 2$ & $36 \pm 4$ & $41 \pm 3$ & $37 \pm 4$ & $37 \pm 5$ \\
\hline Sodium (mEq/liter) & $138 \pm 2$ & $142 \pm 1$ & $146 \pm 1$ & $141 \pm 1$ & $143 \pm 2$ & $141 \pm 8$ \\
\hline Total protein (grams/dl) & $4.7 \pm 0.1$ & $6.0 \pm 0.1$ & $5.1 \pm 0.1$ & $5.0 \pm 0.1$ & $5.2 \pm 0.2$ & $5.6 \pm 0.1$ \\
\hline Triglycerides $(\mathrm{mg} / \mathrm{dl})$ & $57 \pm 8$ & $32 \pm 5$ & $64 \pm 14$ & $55 \pm 6$ & $37 \pm 3$ & $41 \pm 5$ \\
\hline Uric acid (mg/dl) & 0.0 & 0.0 & 0.0 & 0.0 & 0.0 & 0.0 \\
\hline
\end{tabular}

Rabbits were injected intravenously or transfected intraarterially with DNA liposome complexes $(n=17)$ or with saline $(n=6)$. Blood samples were obtained prior to sacrifice, and analyzed (Roche Biomedical Laboratories, Dublin, OH). Results were compared with normal rabbits at the University of Michigan $(n=6)$. Mean values, SEM, and the number of subjects (in parentheses) are shown.

Tissues were analyzed from immune mice in which an immune response to the murine class I MHC gene, $\mathrm{H}-2 \mathrm{~K}^{\mathrm{S}}$, had been generated as a result of direct gene transfer. These mice were compared to control nonimmune mice injected with either human HLA-B7, $\beta$-galactosidase, or in one instance, an $\mathrm{H}-2 \mathrm{~K}^{\mathbf{s}}$ plasmid in which $\mathrm{H}-2 \mathrm{~K}^{\mathrm{S}}$ did not elicit an allogeneic $\mathrm{MHC}$ response. Animals were analyzed for cytolytic T-cell activity against CT26 K $\mathrm{K}^{\mathrm{S}}$ adenocarcinoma cells and/or CT26 cells (negative control), and an immune response to $\mathrm{H}-2 \mathrm{~K}^{S}$ was confirmed in 10 animals (Table 1). When pathologic analyses of tissues from these animals were performed, no evidence of autoimmunity or toxicity was observed despite the documented immune response to $\mathrm{H}-2 \mathrm{~K}^{\mathrm{S}}$ (Table 1). Incidental pathologic findings were noted infrequently that were clinically insignificant and unrelated to expression of $\mathrm{H}-2 \mathrm{~K}^{\mathrm{S}}$. Mild hepatocellular injury was seen in several mice due to mouse hepatitis virus (Jacoby and Fox, 1984), which was diagnosed by serological testing in these mice. Therefore, no adverse effects were associated with this form of therapy in mice at DNA liposome doses that exceed the human dose by $\geqslant 100$-fold.

In additional studies, tissues from a porcine model of vasculitis were examined. These animals were transfected with the human class I MHC gene, HLA-B7, directly into iliofemoral arteries using DNA-liposome complexes (Nabel et al., 1992a). Iliofemoral arteries from animals sacrificed 10 or 17 days after transfection with HLA-B7 DNA liposomes demonstrated an intense inflammatory reaction, consisting of mononuclear cells, primarily in the adventitia. Medial inflammation and intimal thickening were also present. Other systemic organs, including kidney, liver, spleen, lung, and nontransfected carotid artery, were examined to determine whether systemic autoimmunity was present. These organs did not reveal inflammatory changes and were normal in appearance on light microscopy (D.G.). In one pig sacrificed 75 days after transduction of iliofemoral arteries with HLA-B7, a residual healed vasculitis was detected in the iliofemoral arteries although the recombinant HLA-B7 DNA was no longer present by PCR analysis. The lung, kidney, spleen, liver, and carotid artery in this animal showed normal histology.

\section{Cumulative experience with organ toxicity}

To determine whether there is any organ toxicity associated with the use of cationic liposomes for the introduction of recombinant genes, we have summarized our experience in porcine and rabbit studies. Seven plasmids were studied, including the $E$. coli $\beta$-galactosidase gene, the human factor IX gene, the human PDGF gene, the human acidic FGF gene, the human TGF- $\beta$ gene, the human TGF- $\alpha$ gene, and the human HLA-B7 gene. Organs from pigs and rabbits that had undergone direct arterial transfection were analyzed and compared with normal pigs and rabbits, respectively, that had not undergone arterial transfection, for evidence of possible systemic organ toxicity (Tables 2 and 3). Heart, lung, liver, kidney, spleen, skeletal muscle, nontransfected artery, ovary, and testes demonstrated no major immune or inflammatory changes or cytopathology with any of the seven plasmids. Occasional minor abnormalities were observed in both experimental and control animals, 
Table 5. Localization of Plasmid DNA by PCR after Direct Gene Transfer IN Vivo IN PORCINE AND RABBIT MODELS

\begin{tabular}{|c|c|c|c|c|c|c|c|}
\hline Species & $\begin{array}{c}\text { Pig } \\
(\mathrm{n}=6)\end{array}$ & $\begin{array}{c}\text { Pig } \\
(\mathrm{n}=11)\end{array}$ & $\begin{array}{l}\text { Rabbit } \\
(\mathrm{n}=10)\end{array}$ & $\begin{array}{l}\text { Rabbit } \\
(\mathrm{n}=2)\end{array}$ & $\begin{array}{l}\text { Rabbit } \\
(\mathrm{n}=4)\end{array}$ & $\begin{array}{l}\text { Rabbit } \\
(\mathrm{n}=4)\end{array}$ & $\begin{array}{l}\text { Rabbit } \\
(\mathrm{n}=5)\end{array}$ \\
\hline $\begin{array}{l}\text { Site of introduction } \\
\text { Duration }\end{array}$ & $\begin{array}{l}\text { Artery } \\
7 \text { days }\end{array}$ & $\begin{array}{c}\text { Artery } \\
17-21 \text { days }\end{array}$ & $\begin{array}{l}\text { I.V. } \\
3 \text { days }\end{array}$ & $\begin{array}{l}\text { I. V. } \\
35 \text { days }\end{array}$ & $\begin{array}{l}\text { Artery } \\
7 \text { days }\end{array}$ & $\begin{array}{c}\text { Artery } \\
26-30 \text { days }\end{array}$ & $\begin{array}{c}\text { Artery } \\
76-126 \text { days }\end{array}$ \\
\hline Recombinant gene & $a, d, e^{a}$ & $a, b, d, f$ & $b, c$ & $\mathrm{~g}$ & $\mathrm{a}, \mathrm{g}$ & $\mathrm{a}, \mathrm{g}$ & $\mathrm{a}, \mathrm{g}$ \\
\hline \multicolumn{8}{|l|}{ Gonadal tissue } \\
\hline Ovary & $0 / 6$ & $0 / 10$ & $0 / 0$ & $0 / 0$ & $0 / 0$ & $\begin{array}{l}0 / 4 \\
0 / 0\end{array}$ & $\begin{array}{l}0 / 0 \\
0 / 5\end{array}$ \\
\hline Testes & $0 / 0$ & $0 / 1$ & $0 / 10$ & $0 / 1$ & $0 / 3$ & 0/0 & $\begin{array}{r}0 / 5 \\
0 / 2\end{array}$ \\
\hline Heart & $0 / 6$ & $0 / 11$ & $3 / 10$ & ND & ND & ND & ND \\
\hline Lung & $0 / 6$ & $0 / 11$ & $1 / 10$ & $1 / 2$ & $2 / 4$ & $0 / 4$ & $1 / 5$ \\
\hline Liver & $1 / 6$ & $0 / 11$ & $0 / 10$ & $0 / 2$ & $1 / 4$ & $0 / 4$ & $0 / 5$ \\
\hline Kidney & $1 / 6$ & $0 / 11$ & $0 / 10$ & $0 / 2$ & $1 / 4$ & $0 / 4$ & $0 / 5$ \\
\hline Spleen & $1 / 6$ & $0 / 11$ & $0 / 10$ & $0 / 5$ & $3 / 4$ & $0 / 4$ & $0 / 5$ \\
\hline Muscle & $0 / 6$ & $0 / 11$ & $0 / 10$ & ND & ND & ND & ND \\
\hline Brain & ND & ND & $0 / 10$ & ND & ND & ND & ND \\
\hline Transfected artery & $5 / 6$ & $9 / 11$ & None & None & $3 / 5$ & $4 / 4$ & $6 / 6$ \\
\hline Nontransfected artery & $0 / 6$ & $0 / 11$ & $0 / 10$ & $0 / 2$ & $0 / 4$ & ND & $0 / 2$ \\
\hline Negative controls & $0 / 6$ & $0 / 11$ & $0 / 10$ & $0 / 2$ & $0 / 4$ & $0 / 4$ & $0 / 2$ \\
\hline Positive controls & $6 / 6$ & $11 / 11$ & $10 / 10$ & $5 / 5$ & $4 / 4$ & $4 / 4$ & $5 / 5$ \\
\hline
\end{tabular}

DNA-liposome complexes were introduced by the indicated route in a $0.7-\mathrm{ml}$ volume in pigs $(n=17)$ or rabbits $(n=25)$. In the case of intraarterial gene transfer, an attempt was made to remove unreactive DNA-liposome complexes. DNA was extracted from the indicated organs and analyzed by PCR. The number of animals tested positive relative to the total number analyzed is indicated.

ND is not determined.

a a. E. coli $\beta$-galactosidase; b. human HLA-B7; c. murine H-2K ${ }^{\text {; }}$ d. human PDGF B; e. human acidic FGF; f. human TGF- $\alpha$; g. human factor IX.

including focal peribronchial lymphoid aggregates in the lung, focal portal mononuclear inflammatory cells in the liver, focal tubular stasis and interstitial mononuclear inflammatory cells in the kidney, and splenic congestion which were not clinically significant. These incidental findings were unrelated to the introduction of exogenous DNA.

In addition to the analysis of organ toxicity by pathologic analysis, we performed routine analysis of serum enzymes and biochemical parameters that provide a more sensitive biochemical indicator of organ toxicity. Blood samples from pigs transfected with one of the seven plasmids (experimental group) $(n=28)$ were compared to nontransfected animals who underwent sham operative procedures (saline controls) $(n=5)$ and to normal pigs who had no experimental manipulation (normal controls $(n=7)$. These samples revealed no statistical differences in liver function, renal function, glucose, or serum electrolytes between the three groups: experimental group, saline controls, normal controls $(p=$ NS) (Table 3). Similarly, rabbits transduced with one of the seven plasmids (experimental group) $(n=17)$ showed a range of values similar to sham operated saline controls $(n=6)(p=$ NS) and rabbits who had not undergone experimental manipulation (normal controls) $(n=6)(p=$ NS) (Table 4).

\section{Animal studies: lack of recombinant plasmid in gonadal tissue}

To determine whether DNA liposomal transduction allowed uptake of plasmid DNA into gonadal tissue, we performed PCR analysis of transfected tissues and systemic organs. The cumu- lative findings from direct gene transfer using DNA liposome complexes have been summarized from studies of 17 pigs, 25 rabbits, and 17 mice. These animals received DNA-liposome complexes by arterial transfection, intravenous injection, subcutaneous injection, or intraperitoneal injections (Tables 1 and 5). Seven plasmid expression vectors were studied, including $E$. coli $\beta$-galactosidase, human HLA-B7, murine $\mathrm{H}-2 \mathrm{~K}^{\mathrm{s}}$, human PDGF B, human acidic FGF, human TGF- $\alpha$, and human factor IX genes.

In animals transduced by catheter-mediated gene transfer into the arterial wall, evidence of recombinant DNA was consistently observed in transfected, but not in nontransfected, arterial sites by PCR analysis (Table 5 and Fig. 1A,B). In the porcine model, plasmid DNA was detected in kidney, liver, and spleen of one pig following arterial transfection. In other pigs, plasmid DNA was detected only at the site of arterial transfection and was not present in other tissues. When mixtures of positive tissue extracts (arteries) were mixed with negative tissue, the PCR signals were easily detected, suggesting that there are no inhibitors in the tissue extracts (Fig. 1B). In rabbits, it was technically more difficult to remove unreacted DNA-liposome complexes from the vessel after gene transfer, and DNA was occasionally detected in other organs, including heart, lung, liver, kidney, and spleen. Seven days post arterial transfection, recombinant DNA was present in lung, liver, kidney, and spleen of rabbits, but none in gonadal tissue. In rabbits that received a direct intravenous infusion, plasmid DNA was detected by PCR 3 days following gene transfer in heart and lung. Plasmid DNA was not detected in gonadal tissue from the 17 pigs, 25 rabbits, or 17 mice. 

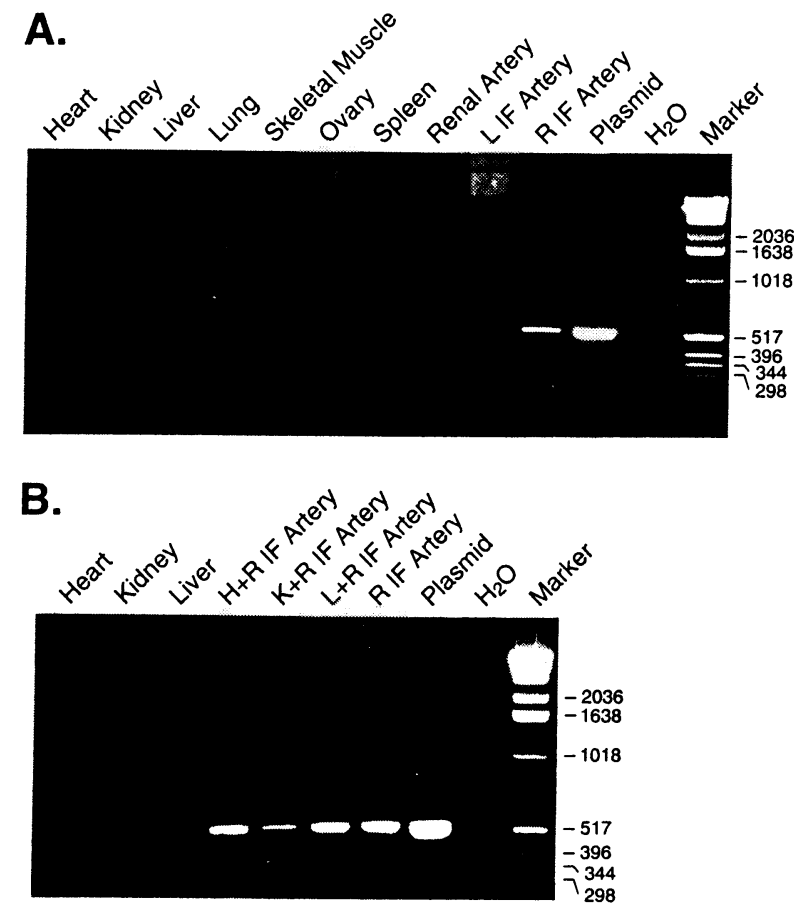

Fig. 1. A. Expression of recombinant human HLA-B7 DNA in porcine tissues in a representative pig following direct arterial gene transfer. Recombinant HLA-B7 plasmid DNA was not detected in heart, kidney, liver, lung, skeletal muscle, ovary, spleen, or nontransfected right renal artery. The presence of recombinant human HLA-B7 DNA in transduced left and right iliofemoral arteries was detected 21 days following direct arterial transfection. Positive controls and negative control are provided in addition to marker DNA. IF, Iliofemoral; L, left; $R$, right. $B$. Lack of inhibitor for the PCR reaction in negative tissues. Human HLA-B7 DNA was not present in porcine heart, kidney, or lung. When mixtures of positive arterial tissues were mixed with negative tissues, PCR signals were still detected in heart plus right iliofemoral artery ( $\mathrm{H}+\mathrm{RIF}$ artery), kidney plus right iliofemoral artery ( $\mathrm{K}+\mathrm{RIF}$ artery), and lung plus right iliofemoral artery (L+RIF artery). Human HLA-B7 DNA is present in transfected right iliofemoral artery. Positive controls and negative controls are shown along with DNA markers.

\section{DISCUSSION}

In this study, three questions have been addressed that are relevant to potential long-term complications of direct gene transfer by DNA liposomes in vivo. The possibility that this method of gene transfer could induce autoimmunity or diffuse toxicity has been analyzed, and the potential for uptake in gonadal tissue has been examined. We have found no evidence of autoimmunity, biochemical abnormalities, or tissue pathology in porcine, rabbit, or murine models. In addition, gonadal tissues did not contain recombinant DNA after direct gene transfer of DNA-liposome complexes in vivo in contrast to some other organs. These findings suggest that this method of gene transfer in vivo poses no readily apparent contraindications to use in humans.

Although the introduction of DNA liposome complexes provided no obvious acute toxicities in previous studies (Stewart $e t$ al., 1992), earlier analyses had not addressed the question whether the introduction of a foreign histocompatibility gene might induce generalized autoimmunity chronically. To some extent, previous studies with organ transplantation provide precedent for this question. A substantial amount of foreign transplantation antigen is introduced in vivo following transplantation, and previous extensive clinical experience suggests that autoimmunity has rarely been observed in association with organ transplantation (for review, see Klumpp et al., 1990; Goldman et al., 1991). Although direct gene transfer poses a different clinical setting, the previous transplantation experience suggested that transfer of foreign MHC genes per se did not induce autoimmunity. In these studies, we find that direct gene transfer of foreign MHC induces no detectable immunoreactivity against normal tissues, despite the successful generation of an immune response to the foreign haplotype (Table 1).

Finally, the concern has been raised about the potential for inadvertent introduction of DNA into germ-line cells following direct gene transfer. To address this issue, we examined the ovaries and testes from pigs, rabbits, and mice following the delivery of DNA liposomes by intravenous injection or intraarterial transfection. PCR analysis revealed no evidence of uptake or expression in gonadal tissue. Although we cannot exclude that it may occur at lower frequency or in selected clinical settings, these data suggested that germ-line gene transfer is unlikely to occur frequently using this method of gene transfer.

The potential of gene therapy to contribute to the treatment of human diseases has expanded significantly in recent years. Advances in gene transfer techniques have improved the prospects for novel approaches to the delivery of recombinant genes in vivo. In addition to their role in gene therapy, these gene transfer approaches can also be used to address important mechanistic questions in disease (Nabel et al., 1992b). Although direct gene transfer technology remains in its infancy, expanding laboratory efforts and the lack of apparent toxicity from this form of treatment suggest that it will become increasingly useful for the genetic treatment of human disease.

\section{ACKNOWLEDGMENTS}

We gratefully acknowledge Diane Smith and Linda Looby for their assistance in the animal procedures, John DeCoster for providing excellent care of the animals, and Mrs. Gail Reisdorph and Betty Plunkett for manuscript preparation.

\section{REFERENCES}

ARRICK, B.A., LOPEZ, A.R., ELFMAN, F., EBNER, R., DAMSKY, C.H., and DERYNCK, R. (1992). Altered metabolic and adhesive properties and increased tumorigenesis associated with increased expression of transforming growth factor $\beta 1$. J. Cell Biol. 118, 715-726.

CORNETTA, K., MOEN, R.C., CULVER, K., MORGAN, R.A., MCLACHLIN, J.R., STURM, S., SELEGUE, J., LONDON, W., BLAESE, R.M., and ANDERSON, W.F. (1990). Amphotropic murine leukemia retrovirus is not an acute pathogen for primates. Hum. Gene Ther. 1, 15-30. 
CORNETTA, K., MORGAN, R.A., and ANDERSON, W.F. (1991). Safety issues related to retroviral-mediated gene transfer in humans. Hum. Gene Ther. 2, 5-14.

FEARON, E.R., PARDOLL, D.M., ITAYA, T., GOLUMBEK, P., LEVITSKY, H.I., SIMONS, J.W., KARASUYAMA, H., VOGELSTEIN, B., and FROST, P. (1990). Interleukin-2 production by tumor cells bypasses $T$ helper function in the generation of an antitumor response. Cell 60, 397-403.

FELGNER, P.L., and RHODES, G. (1991). Gene therapeutics. Nature 349, 351-352.

FELGNER, P.L., GADEK, T.R., HOLM, H., ROMAN, R., CHAN, H.W., WENZ, M. NORTHROP, J.P., RINGOLD, G.M., and DANIELSEN, M. (1987). Lipofection: a highly efficint, lipid-mediated DNA-transfection procedure. Proc. Natl. Acad. Sci. USA 84, 7413-7417.

GAO, X., and HUANG, L. (1991). A novel cationic liposome reagent for efficient transfection of mammalian cells. Biochem. Biophys. Res. Commun. 179, 280-285.

GOLDMAN, M., DRUET, P., and GLEICHMANN, E. (1991). $T_{H} 2$ cells in systemic autoimmunity: insights from allogeneic diseases and chemically-induced autoimmunity. Immunol. Today 12, 223-227.

JACOBY, R.O., and FOX, J.G. (1984). Biology and diseases of mice. In Laboratory Animal Medicine. J.G. Fox, B.J. Cohen, and F.M. Lowe, eds. (Academic Press, New York).

KLUMPP, T.R., CALIGURI, M.A., RABINOWE, S.N., SOIFFER, R.J., MURRAY, C., and RITZ, J. (1990). Autoimmune pancytopenia following allogeneic bone marrow transplantation. Bone Marrow Transplant. 6, 445-457.

LEMAN, A.D., STRAW, B., GLOCK, R.D., MENGELING, W.L., PENNY, R.H.C., and SCHOLL, E. (1986). Hematology and clinical chemistry. In Diseases of Swine. (Iowa State University Press, Ames, lowa) pp. 29-40.

LIM, C.S., CHAPMAN, G.D., GAMMON, R.S., MUHLESTEIN, J.B., BAUMAN, R.P., STACK, R.S., and SWAIN, J.L. (1991). Direct in vivo gene transfer into the coronary and peripheral vasculatures of the intact dog. Circulation 83, 2007-2011.

NABEL, E.G., PLAUTZ, G., BOYCE, F.M., STANLEY, J.C., and NABEL, G.J. (1989). Recombinant gene expression in vivo within endothelial cells of the arterial wall. Science 244, 1342-1344.

NABEL, E.G., PLAUTZ, G., and NABEL, G.J. (1990). Site-specific gene expression in vivo by direct gene transfer into the arterial wall. Science 249, 1285-1288.

NABEL, E.G., PLAUTZ, G.E., and NABEL, G.J. (1992a). Expres- sion of a foreign histocompatibility gene in the arterial wall induces focal vasculitis in vivo. Proc. Natl. Acad. Sci. USA 89, 5157-5161. NABEL, G.J., YANG, Z., DERYNCK, R., HAUDENSCHILD, C., MACIAG, T., and NABEL, E.G. (1992b). Analysis of vessel function by direct gene transfer in vivo. J. Cell. Biochem. 16a, 3.

PLAUTZ, G.E., YANG, Z.Y., GAO, X., HUANG, L., and NABEL, G.J. (1992). Immunotherapy of malignancy by in vivo gene transfer into tumors. (submitted).

PRICE, J., TURNER, D., and CEPKO, C. (1987). Lineage analysis in the vertebrate nervous system by retrovirus-mediated gene transfer. Proc. Natl. Acad. Sci. USA 84, 156-160.

ROMANO, P.J. (1986). Cytolytic methods. In Manual of Clinical Laboratory Immunology. N.R. Rose and H. Friedman, eds. (American Society for Microbiology, New York).

ROSENBERG, S.A., AEBERSOLD, P., CORNETTA, K., KASID, A., MORGAN, R.A., MOEN, R., KARSON, E.M., LOTZE, M.T., YANG, J.C., TOPALIAN, S.L., MERINO, M.J., CULVER, K., MILLER, A.D., BLAESE, R.M., and ANDERSON, W.F. (1990). Gene transfer into humansimmunotherapy of patients with advanced melanoma, using tumor-infiltrating lymphocytes modified by retrovial gene transduction. N. Engl. J. Med. 323, 570-578.

STEWART, M.J., PLAUTZ, G.E., DEL BUONO, L., YANG, Z.Y., XU, L., GAO, X., HUANG, L., NABEL, E.G., and NABEL, G.J. (1992). Gene transfer in vivo with DNA-liposome complexes: safety and acute toxicity in mice. Hum. Gene Ther. 3, 267-275.

YAO, S.Y., WILSON, J.M., NABEL, E.G., KURACHI, S., HACHIYA, H.L., and KURACHI, K. (1991). Expression of human factor IX in rat capillary endothelial cells: toward somatic gene therapy for hemophilia B. Proc. Natl. Acad. Sci. USA 88, 81018105.

\author{
Address reprint requests to: \\ Dr. Elizabeth G. Nabel \\ University of Michigan \\ MSRB II, Room 3560 \\ 1150 W. Medical Center Drive \\ Ann Arbor, MI 48109-0688
}

Received for publication June 29, 1992; accepted after revision September 17, 1992. 


\section{This article has been cited by:}

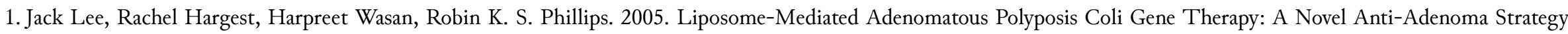
in Multiple Intestinal Neoplasia Mouse Model. Diseases of the Colon \& Rectum 47:12, 2105-2113. [CrossRef]

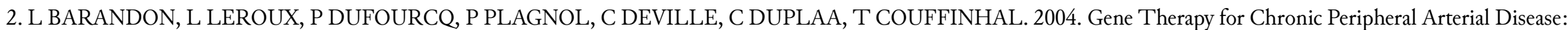
What Role for the Vascular Surgeon?. Annals of Vascular Surgery 18:6, 758-765. [CrossRef]

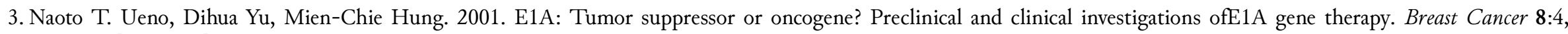
285-293. [CrossRef]

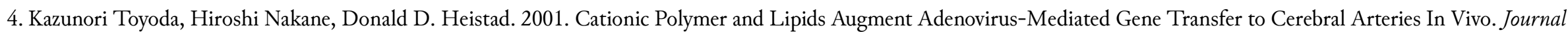
of Cerebral Blood Flow \& Metabolism 1125-1131. [CrossRef]

5. I Baumgartner, JM Isner. 2001. SOMATIC GENE THERAPY IN THE CARDIOVASCULAR SYSTEM. Annual Review of Physiology 63:1, 427-450. [CrossRef]

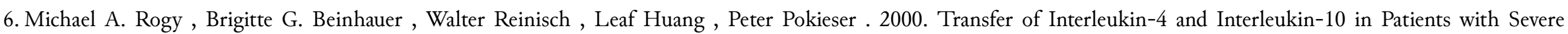

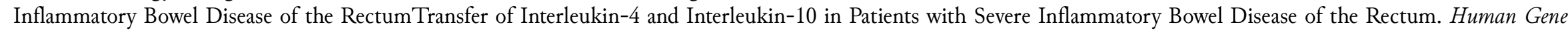
Therapy 11:12, 1731-1741. [Abstract] [PDF] [PDF Plus]

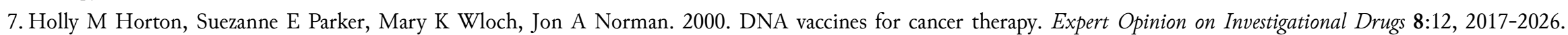
[CrossRef]

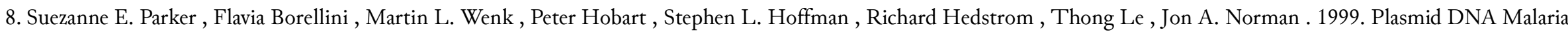

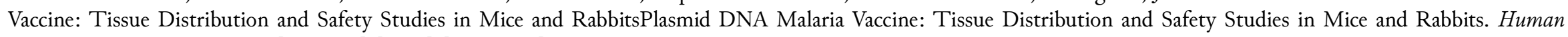
Gene Therapy 10:5, 741-758. [Abstract] [PDF] [PDF Plus]

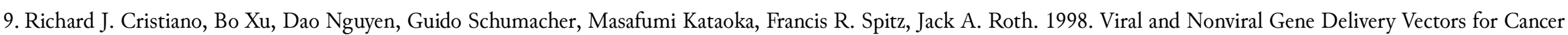
Gene Therapy. Cancer Detection <btml_ent glyph="@amp;" ascii="Jamp;"/> Prevention 22:5, 445-454. [CrossRef]

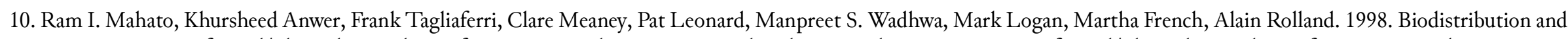

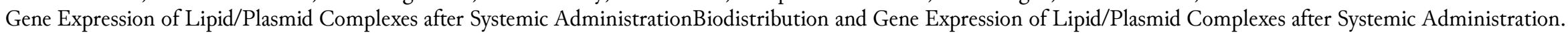
Human Gene Therapy 9:14, 2083-2099. [Abstract] [PDF] [PDF Plus]

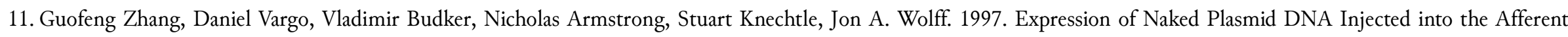

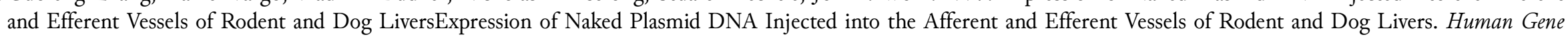
Therapy 8:15, 1763-1772. [Abstract] [PDF] [PDF Plus]

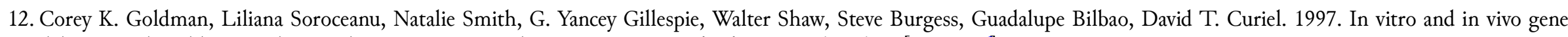
delivery mediated by a synthetic polycationic amino polymer. Nature Biotechnology 15:5, 462-466. [CrossRef]

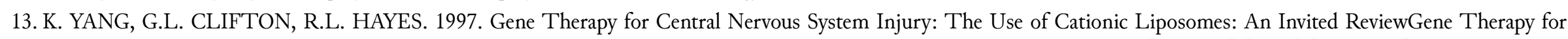
Central Nervous System Injury: The Use of Cationic Liposomes: An Invited Review. Journal of Neurotrauma 14:5, 281-297. [Abstract] [PDF] [PDF Plus]

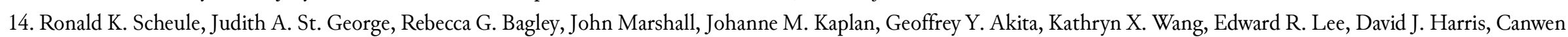

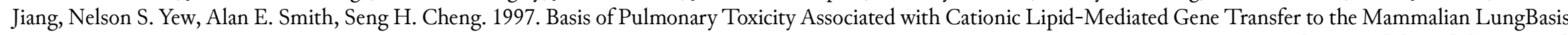

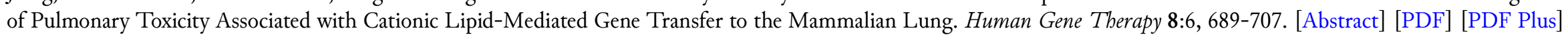


15. Koicni Takakuwa, Kazuyuki Fujita, Akira Kikuchi, Susumu Sugaya, Tetsuro Yahata, Hiroshi Aida, Takumi Kurabayashi, Isao Hasegawa, Kenichi Tanaka. 1997. Direct Intratumoral Gene Transfer of the Herpes Simplex Virus Thymidine Kinase Gene with DNA-liposome Complexes: Growth Inhibition of Tumors and Lack of Localization in Normal Tissues. Cancer Science 88:2, 166-175. [CrossRef]

16. M. Xu, D. Kumar, S. Srinivas, L. J. Detolla, S. F. Yu, S. A. Stass, A. J. Mixson. 1997. Parenteral Gene Therapy with p53 Inhibits Human Breast Tumors In Vivo Through a Bystander Mechanism Without Evidence of ToxicityParenteral Gene Therapy with p53 Inhibits Human Breast Tumors In Vivo Through a Bystander Mechanism Without Evidence of Toxicity. Human Gene Therapy 8:2, 177-185. [Abstract] [PDF] [PDF Plus]

17. R. A. Winegar, J. A. Monforte, K. D. Suing, K. G. O'loughlin, C. J. Rudd, J. T. MacGregor. 1996. Determination of Tissue Distribution of an Intramuscular Plasmid Vaccine Using PCR and In Situ DNA HybridizationDetermination of Tissue Distribution of an Intramuscular Plasmid Vaccine Using PCR and In Situ DNA Hybridization. Human Gene Therapy 7:17, 2185-2194. [Abstract] [PDF] [PDF Plus]

18. Jonathan Fox. 1996. Therapeutic Drug Monitoring 18:4, 410-422. [CrossRef]

19. Marion Baudard, Terence R. Flotte, Josep M. Aran, Alain R. Thierry, Ira Pastan, Myung-Geol Pang, William G. Kearns, Michael M. Gottesman. 1996. Expression of the Human Multidrug Resistance and Glucocerebrosidase cDNAs from Adeno-Associated Vectors: Efficient Promoter Activity of AAV Sequences and In Vivo Delivery via LiposomesExpression of the Human Multidrug Resistance and Glucocerebrosidase cDNAs from Adeno-Associated Vectors: Efficient Promoter Activity of AAV Sequences and In Vivo Delivery via Liposomes. Human Gene Therapy 7:11, 1309-1322. [Abstract] [PDF] [PDF Plus]

20. PRINCIPAL INVESTIGATOR: Jeffrey M. Isner, CO-PRINCIPAL INVESTIGATOR: Ken Walsh, CO-INVESTIGATORS: James Symes, Ann Pieczek, Satoshi Takeshita, Jason Lowry, Kenneth Rosenfield, Lawrence Weir, Edi Brogi, Dan Jurayj. 1996. Arterial Gene Transfer for Therapeutic Angiogenesis in Patients with Peripheral Artery Disease. St. Elizabeth's Medical Center, Tufts University School of Medicine, Boston, MassachusettsArterial Gene Transfer for Therapeutic Angiogenesis in Patients with Peripheral Artery Disease. St. Elizabeth's Medical Center, Tufts University School of Medicine, Boston, Massachusetts. Human Gene Therapy 7:8, 959-988. [Abstract] [PDF] [PDF Plus]

21. CHARLES COUTELLE, ROBERT WILLIAMSON. 1996. Liposomes and Viruses for Gene Therapy of Cystic FibrosisLiposomes and Viruses for Gene Therapy of Cystic Fibrosis. Journal of Aerosol Medicine 9:1, 79-88. [Abstract] [PDF] [PDF Plus]

22. RONALD L. HAYES, KEYI YANG, JANET S. WHITSON, RAND POSMANTUR. 1995. Cytoskeletal Derangements Following Central Nervous System Injury: Modulation by Neurotrophic Gene TransfectionCytoskeletal Derangements Following Central Nervous System Injury: Modulation by Neurotrophic Gene Transfection. Journal of Neurotrauma 12:5, 933-941. [Abstract] [PDF] [PDF Plus]

23. Fred D. Ledley. 1995. Nonviral Gene Therapy: The Promise of Genes as Pharmaceutical ProductsNonviral Gene Therapy: The Promise of Genes as Pharmaceutical Products. Human Gene Therapy 6:9, 1129-1144. [Abstract] [PDF] [PDF Plus]

24. J. GEOFFREY PICKERING, SATOSHI TAKESHITA, LAURENT FELDMAN, JEFFREY M. ISNER. 1995. Vascular Applications of Human Gene Therapy. Journal of Interventional Cardiology 8:4, 373-376. [CrossRef]

25. Denise Lew, Suezanne E. Parker, Terrie Latimer, Anna M. Abai, Ann Kuwahara-Rundell, Soeun Grace Doh, Zhi-Yong Yang, Drake Laface, Stanislaw H. Gromkowski, Gary J. Nabel, Marston Manthorpe, Jon Norman. 1995. Cancer Gene Therapy Using Plasmid DNA: Pharmacokinetic Study of DNA Following Injection in MiceCancer Gene Therapy Using Plasmid DNA: Pharmacokinetic Study of DNA Following Injection in Mice. Human Gene Therapy 6:5, 553-564. [Abstract] [PDF] [PDF Plus]

26. Suezanne E. Parker, H. Lee Vahlsing, Laurie M. Serfilippi, Craig L. Franklin, Soeun G. Doh, Stanislaw H. Gromkowski, Denise Lew, Marston Manthorpe, Jon Norman. 1995. Cancer Gene Therapy Using Plasmid DNA: Safety Evaluation in Rodents and Non-Human PrimatesCancer Gene Therapy Using Plasmid DNA: Safety Evaluation in Rodents and Non-Human Primates. Human Gene Therapy 6:5, 575-590. [Abstract] [PDF] [PDF Plus] 
27. Leslie A. Lesoon-Wood, Woo Ho Kim, Hynda K. Kleinman, Bruce D. Weintraub, A. James Mixson. 1995. Systemic Gene Therapy with p53 Reduces Growth and Metastases of a Malignant Human Breast Cancer in Nude MiceSystemic Gene Therapy with p53 Reduces Growth and Metastases of a Malignant Human Breast Cancer in Nude Mice. Human Gene Therapy 6:4, 395-405. [Abstract] [PDF] [PDF Plus]

28. Natasha J. Caplen, Eric W.F.W. Alton, Peter G. Mddleton, Julia R. Dorin, Barbara J. Stevenson, Xiang Gao, Stephen R. Durham, Peter K. Jeffery, Margaret E. Hodson, Charles Coutelle, Leaf Huang, David J. Porteous, Robert Williamson, Duncan M. Geddes. 1995. Liposome-mediated CFTR gene transfer to the nasal epithelium of patients with cystic fibrosis. Nature Medicine 1:1, 39-46. [CrossRef]

29. Elizabeth G. Nabel, Zhiyong Yang, David Muller, Alfred E. Chang, Xiang Gao, Leaf Huang, Kyung J. Cho, Gary J. Nabel. 1994. Safety and Toxicity of Catheter Gene Delivery to the Pulmonary Vasculature in a Patient with Metastatic MelanomaSafety and Toxicity of Catheter Gene Delivery to the Pulmonary Vasculature in a Patient with Metastatic Melanoma. Human Gene Therapy 5:9, 1089-1094. [Abstract] [PDF] [PDF Plus]

30. Nancy Sajjadi, Edgar Kamantigue, Wilson Edwards, Tammi Howard, Doug Jolly, Steven Mento, Sunil Chada. 1994. Recombinant Retroviral Vector Delivered Intramuscularly Localizes to the Site of Injection in MiceRecombinant Retroviral Vector Delivered Intramuscularly Localizes to the Site of Injection in Mice. Human Gene Therapy 5:6, 693-699. [Abstract] [PDF] [PDF Plus]

31. GREGORY E. PLAUTZ, ELIZABETH G. NABEL, BERNARD FOX, ZHI-YONG YANG, MICHELE JAFFE, DAVID GORDON, ALFRED CHANG, GARY J. NABEL. 1994. Direct Gene Transfer for the Understanding and Treatment of Human Disease. Annals of the New York Academy of Sciences 716:1 Gene Therapy, 144-153. [CrossRef]

32. HASSAN FARHOOD, XIANG GAO, KYONGHEE SON, JOHN S. LAZO, LEAF HUANG, JAMES BARSOUM, REMO BOTTEGA, RICHARD M. EPAND. 1994. Cationic Liposomes for Direct Gene Transfer in Therapy of Cancer and Other Diseases. Annals of the New York Academy of Sciences 716:1 Gene Therapy, 23-35. [CrossRef]

33. Hong San, Zhi-Yong Yang, Vincent J. Pompili, Michele L. Jaffe, Gregory E. Plautz, Ling Xu, Jiin H. Felgner, Carl J. Wheeler, Philip L. Felgner, Xiang Gao, Leaf Huang, David Gordon, Gary J. Nabel, Elizabeth G. Nabel. 1993. Safety and Short-Term Toxicity of a Novel Cationic Lipid Formulation for Human Gene TherapySafety and Short-Term Toxicity of a Novel Cationic Lipid Formulation for Human Gene Therapy. Human Gene Therapy 4:6, 781-788. [Abstract] [PDF] [PDF Plus] 